\title{
Traumatic Brain Injury: A Study of the Qualitative Aspect of Brain Injury Biomechanics
}

\author{
*Mahvash Afzal ${ }^{1}$, Khalid Zaidi ${ }^{2}$ \\ Dept. of Mechanical Engineering, Z.H. College of Engineering and Technology, Aligarh Muslim University, \\ Aligarh, India
}

\begin{abstract}
This paper aims to provide an overview of the literature on the mechanics and diagnosis of traumatic brain injuries (TBI). The understanding of TBI is of prime importance for diagnosis and subsequent treatment and rehabilitation of those affected. The mechanism of a brain injury may be any of the following: head acceleration-deceleration,thoracic pressure surge, anddirect transmission through cranial bone. Researchers have used experimental methods such as shock generation via shock tube to simulate trauma conditions and computational tools such as Finite Element Modeling tomodel the brain tissue and analyze the effects of various brain injury mechanisms. Through the study of the literature available an attempt has been made to gain an insight into the qualitative aspect of mechanics of traumatic brain injuries and to consolidate the findings so far, in order to assist the diagnosis and treatment.
\end{abstract}

Keywords: traumatic brain injury, biomechanics, diffused axonal injuries

\section{Introduction}

Biomechanical analysis involves the investigation of an organ as a system subjected to the application of an input in the form of force or impact to the system, and determination of the output using computational, analytical and numerical techniques. The application of biomechanics in conjunction with medical science has resulted in a better understanding of conditions especially those involving trauma, leading to improved preventive, diagnostic, therapeutic and rehabilitative tactics. Traumatic brain injuries can be classified into three types: head acceleration-deceleration, thoracic pressure surge and direct cranial transmission. Brain injuries may also be categorized as focal and diffused. Focal injuries include contusions, lacerations and haematoma while diffused axonal injuries (DAI) are those where the damage is not focal but is widespread over a larger area of the brain.In this article the focus will be on the diffused type of injuries.

If a high pressure wave impacts against the head, it may cause it to jolt-head acceleration and deceleration. The brain moves in the forward direction and immediately a reversal of direction takes place due to the stretching of the soft spinal tissue. Depending upon how the impact occurred, the head starts its movement while the brain resists, thereby, leaving a space in the skull. As this force progresses a centrifugal force lifts the brain. Both inertia and centrifugal force causes the brain to hit the skull. This impact may cause damage to the brain. In case of thoracic mechanism of TBI, a surge of blood to the brain results in increased intracranial pressure (ICP) causing the brain to hit the skull. It may also result in rupturing of the blood vessels of the brain resulting in hematoma. Finally, direct transmission through cranial bone is the type of brain injury in which the pressure wave directly enters into the cranium. It was observed that in rats, the skull being thin allowed the blast waves to pass unchanged through the skull whereas through the skull of pigs two-thirds of the blast wave was seen to pass. Thoracic pressure surge and direct cranial entry is found mainly in context of blast induced Traumatic brain injuries or bTBI.

\section{Physics of Traumatic Brain Injuries \\ 1.1 Acceleration-Deceleration Mechanism}

In traffic or sports accidents or cases of violence, impact may lead to acceleration-deceleration of the head leading to a coup-contrecoup. Coup-contrecoup is a term applied to head injurieswhereby damage is located both at the site of impact and on the opposite side of the head. In such cases the skull is accelerated first (at this point the brain immediately subjacent to the point of impact may be damaged - so called coup injury) and then it moves away from it. As the skull stops, the brain then impacts on the internal surface of the skull which has bony protuberances, resulting in damage to brain tissue, that is contrecoup.

According to Holbourn, damage to the brain due to impactoccurs as a result of the movements, forces and deformations at each point in the brain(Holbourn, 1940). The movements, forces and deformations are all dependent on the impact; therefore it is sufficient to express everything in terms of deformations. Skull and brain motion and the forces causing them are governed by Newton's Second Law of Motion. The displacement of the head can be described as planar or as occurring in 3-dimensional space. When the skull is moved in a straight line, it is the brain's compressibility which causes it to move. This being very high, the brain moves as a 
whole, and there is no substantial distortion. On the other hand, when the skull is rotated, it is the rigidity of the brain that causes it to move. But its rigidity is small, therefore distortion of a considerable degree is observed. This finding of Holbourn is corroborated analytically and numerically by Bradshaw (Bradshaw and Morfey, 2001). Bradshaw states that the brain has a high bulk modulus leading to negligible volumetric strains or dilatations in the brain tissue upon the application of pressure. However, the relatively low shear modulus is indicative of the development of considerable shear strains or distortions leading to injuries. Thus, it is the distortions that are responsible for injuries and not the dilatations. Therefore, it can be said that an impact which leads to the rotation of the brain has a higher potential to cause damage to the brain tissue.

Goggio hasalso discussed the means by which contrecoup injury may be explained (Goggio, 1941). The passage of a compression wave through the skull has been hypothesized. When the skull is hit, the area hit is deformed or pushed inward on the underlying cortex and a wave of compression passes through the brain tissue toward the opposite side of the skull. When the compression wave reaches the cells of the cortex at the opposite side, these cells are suddenly thrown upon a rigid surface, the skull, hence are damaged by the impact. The skull is modelled as an incompressible shell, filled with semifluidbrain tissue and the impact is modelled as free fall whichis arrested by a resistant object. At the instant of impact, the brain loses the momentum, which was possessed by it due to its mass and velocity of impact. This change in momentum is equal to the reaction force applied on it by the surface over which it falls. This results in the development of a pressure gradient through the brain. A pressure imbalance is momentarily set up and the small blood vesselsin the area of contrecoup are expanded and ruptured by a sudden release ofexternal pressure which is not completely met by a fall in intravascular pressuredue to continuous circulation from the heart.Thus, in addition to brain tissue injury, a haematoma may result from rupturing of the blood vessels in case of coup, contrecoup injuries.

Goldsmith and Plunkett have treatedacceleration-deceleration brain injuries differently depending upon whether they are caused due to impulsive loading characteristics or impact loading (Goldsmith and Plunkett, 2004). The two may occur in succession but not simultaneously. In case of impulsive loading the brain moves as a result of motion imparted to any other part of the body whereas in case of impact loading the impact acts directly on the head. For an unsupported head impulsive loading will cause rotation of the head about the spine. Since the skull and the brain are not rigidly linked they will move at a different rate with respect to each other. This relative motion may produce tensile failure of the bridging veins, which occurs on the average when they are stretched 30\% beyond unloaded length (Lee 1989, Lowenhielm 1974).Impact loading is mechanically different from impulsive loading. It can cause damage to the contact surfacei.e. the scalp and the skull. It may also result in the transmission and reflection of pressure waves in the system as a result of which the phenomena of countercoup and cavitation occur.Contrecoup lesions follow from the bouncing of the brain againstthe inner posterior surface of the skull and the possible developmentof cavitation bubbles within the brain due to high negativepressures. The traveling stress wave reflects against the skull at the contrecoup site and then moves back toward the interior of the parenchyma. It is then followed by a tensile "tail" (negative pressure wave), which produces irreversible cavitation damage in different brain regions, especially within the contrecoup region. Cavitation initiates when the traveling tensile stress reaches a threshold value Pc (critical cavitation pressure $=$ $4.6 \mathrm{kPa}$ at $\left.30^{\circ} \mathrm{C}\right)(\mathrm{Cengel}$ and Boles, 2006), resulting in the formation of bubbles of the cerebro-spinal fluid and an unstable growth of voids in the tissue. This phenomenon is locally amplified by the superposition of primary and reflected tensile waves (ElSayed et al, 2008). The inception of the cavitation theory occurred in 1958 as a result of the attempts made by Gross to explain the pathogenesis ofcontre-coup contusions, occurring on the opposite site of the impact (Gross, 1958). Based on fluid physics, it wassuggested that negative pressures generated on the brain surface on the opposite site of the impactcould rise to magnitudes sufficient to form gas bubbles. Their violent collapse would then lead toparenchyma damage. Based on a physical head model, Lubbock and Goldsmith showed that wavepropagation and reflection processes could indeed provoke cavitation in the intracranial fluids, such asthe cerebrospinal fluid layer, but not in the proper brain tissue (Lubock and Goldsmith, 1980).Lubock and Goldsmith studied the occurrence of cavitation in thelow pressure zones created after impact using both simple spherical shells and cadaver skull replicas. They observed a number of interesting forms of cavitation. Though cavitation was observed to occur with water, it was not observed when gelatin was used. Consequently, the extent to which cavitation magnifies the damage in head injuries depends upon the properties of the cerebro-spinal fluid. Goeller et al simulated blast induced brain trauma using blast wave generated by an 18-inch diameter shock tube. The skull was modelled as an ellipsoid filled with degassed water in one case and Sylgard gel in another case. The presence of cavitation bubbles at the contrecoup in both ellipsoid models was established. In all tests, cavitation at the contrecoup was observed to occur during phases of negative pressure. Collapse of the cavitation bubbles caused by the surrounding pressure and elastic rebound of the skull resulted in significant pressure spikes in the simulated CSF, resulting in a pressure inequity (Goeller et al, 2012). 
From the above discussion it may be deduced that the treatment of a brain trauma resulting from head acceleration-deceleration, depends upon whether the trajectory traced by the skull after impact is translational or curvilinear. In the former case, the mechanism proposed by Goggio may provide better insight into the mechanism of the injury while in the latter case the mechanism proposed by Holbourn and Bradshaw. However, in case of impact loading situations the possibility of cavitation injury has to be kept open. Further insight is needed into the mechanism of cavitation in trauma caused by other than blasts since some researchers such as Ommaya et al in their research carried out in 1971 have ruled out the existence of cavitation as a mechanism of injury deserving much attention (Ommaya, 1971). Researchers have developed instrumentations such as the accelerometers fitted into helmets used by sports personnel which register the acceleration at the time of impact. This has helped to come up with a head injury criterion (HIC), a threshold of acceleration which can cause severe trauma. However, as this study deals with the qualitative aspect of brain injuries, the HIC will only suffice a mention.

\subsection{Thoracic Pressure Surge}

The evidence of thoracic pressure surge as a mechanism for brain injury has been found in ballistic and blast wave injuries to the thorax. The primary factor that distinguishes the thoracic mechanism from other mechanisms of brain injury is that in the former case the ballistic pressure waves originate in the thorax (Courtney and Courtney, 2011). The thorax of human beings or for that matter of any animal is composed of organs having high air and fluid content. If the explosion shock wave hits the thorax, a compression wave is transmitted resulting in barotrauma. For e.g. if the shock wave hits the lungs causing compression of the lungs, it can send a subsequent surge of blood to the brain. As the shock front interacts with the body surface and compresses the abdomen and chest, it transfers its kinetic energy to the body's fluid phase. The resulting hydraulic interaction initiates oscillating waves that traverse the body at about the speed of sound in water and deliver the kinetic energy of the blast wave to the brain. Knudsen and Oen studied the killing of minke whales in Norway by detonation (Knudsen and Oen, 2003). The detonation is achieved with the help of a grenade tipped harpoon, which is aimed at the thorax of the whale. The grenade detonates $60-70 \mathrm{~cm}$ within the body of the whale. Fatal neurotrauma was observed in the whales thus proving that thoracic pressure surge does exist as a mechanism of brain injury caused due to ballistics and blast waves. If the mechanism is true for ballistics then it will be applicable to blast waves also because in essence the two are similar. Thoracic pressure surge was also observed in the abdominal cavity in vehicle crashes. Tests with human volunteers showed that compression up to $20 \%$ during moderate-duration loadingwas fully reversible (Vianno, 1988). Internal organ injury and rib fracture was observed as the compression increased to $40 \%$. It was observed by Lau et al that the speed of loading could change the effect of compression. A high speed loading could result in a compression of even $16 \%$ to result in critical injury. However, whether the thoracic pressure surge can be transmitted to the brain is still unclear as in case of such high impact loading other major organs are likely to suffer extensive damage.

\subsection{Direct Cranial Entry}

This type of brain injury constitutes local distortion of the skull caused by the direct propagation of stress waves through the brain from the point of impact. The skull is not exactly rigid, so the pressure actually deforms the skull as the blast wave moves across. The flexure drives a stress wave that propagates into the brain. The waves flex the skull by only about the width of a human hair. But even that is enough to generate pressures in the brain comparable to an impact. Moss et al, by carrying out hydrodynamic simulations have shown that even a low intensity blast wave at a pressure of as low as 1 bar causes skull flexure comparable to an impact capable of causing injury (Moss et al, 2009). A simplified model of the face consisting of an ellipsoid containing a viscoelastic brain surrounded by CSF was formulated and studied using an arbitrary Eularian-Langragian finite element hydrocode. Due to the viscoelastic properties of the brain tissue surface pressure gets transmitted and the ICP increases. The blast wave transmission across the cranium occurs in just a fraction of a second, leaving no time for the dissipation of pressure. This creates a localized region of very high pressure. Blast waves have been noted to pass through the thin cranium of rats almost unchanged (Chavko et al., 2007) and through the thicker cranium of pigs maintaining about two-thirds magnitude (Bauman et al., 2009). Sundaramurthy et al in their study of effect of Animal Placement Location(APL) on loading condition of the head concluded that the main loading pathways for the rat head are the eye socket and the skull; the snout does not play a major role in loading the brain (Sundaramurthy, 2012).

\section{Conclusions}

After an in-depth study of the qualitative analysis of the mechanics of brain injuries, the following points may be inferred which are expected to aid the classification, diagnosis and treatment of trauma induced brain injuries: 
- Acceleration-deceleration mechanism and direct cranial entry of shock wave seem to be more common and hence more studied mechanisms of brain injury. The thoracic pressure surge as a mechanism for brain trauma has been analysed mainly for blast and ballistic induced injuries only.

- The study of acceleration-deceleration mechanism has revealed rotational acceleration of the brain results in injuries far more significant than those caused as a result of translational acceleration.

- Cavitation produced as a result of acceleration of the brain needs to be explored further as a mechanism for brain injury. It was observed that cavitation occurs in CSF but may not occur in the tissue.

- Thoracic pressure surge was observed to be a proven mechanism in case of bTBI, however whether the thoracic compression wave can be potent enough to damage the brain in sports or vehicular accidents has not been explored sufficiently, primarily because if the wave is potent enough to reach the brain it will cause critical injuries to the thoracic organs such as lungs, heart etc. rendering diagnosis of brain injuries futile.

- Direct cranial entry, like thoracic pressure surge was a mechanism of injury important in context bTBI. It was seen that apart from causing skull flexure (which may occur in any situation of impact), blast waves can reach the brain through orbital and aural openings.

\section{References:}

[1]. Bauman, Richard A., et al. "An introductory characterization of a combat-casualty-care relevant swine model of closed head injury resulting from exposure to explosive blast." Journal of neurotrauma 26.6 (2009): 841-860.

[2]. Bradshaw, D. R. S., and C. L. Morfey. Pressure and shear responses in brain injury models. No. 2001-06-0197.SAE Technical Paper, 2001.

[3]. Cengel and Boles, Thermodynamics: An Engineering Approach, Mc-Graw Hill, 2006

[4]. Chavko M, Koller WA, Prusaczyk WK, McCarronRM. "Measurement of blast wave by a miniature fiberoptic pressure transducer in the rat brain".JNeurol Meth2007;159:277-281.

[5]. Courtney, Michael W., and Amy C. Courtney. "Working toward exposure thresholds for blast-induced traumatic brain injury: thoracic and acceleration mechanisms." Neuroimage 54 (2011): S55-S61.

[6]. El Sayed, Tamer, et al. "Biomechanics of traumatic brain injury." Computer Methods in Applied Mechanics and Engineering 197.51 (2008): 4692-4701

[7]. Holbourn, A. H. S. "Mechanics of head injuries." The Lancet 242.6267 (1943): 438-441.

[8]. Goggio, A. F. "The mechanism of contre-coup injury." Journal of neurology and psychiatry 4.1 (1941): 11.

[9]. Goldsmith, Werner, and John Plunkett."A biomechanical analysis of the causes of traumatic brain injury in infants and children." The American journal of forensic medicine and pathology 25.2 (2004): 89-100.

[10]. Gross AG. A new theory on the dynamics of brain concussion and brain injury.JNeurosur. Sep

[11]. 1958;15(5):548-561.

[12]. Knudsen, Siri K., and Egil O. Øen."Blast-induced neurotrauma in whales."Neuroscience research 46.3 (2003): $377-386$.

[13]. Lau, Veng-Kin, and David C. Viano. "Influence of impact velocity on the severity of nonpenetrating hepatic injury." Journal of Trauma-Injury, Infection, and Critical Care 21.2 (1981): 115-123.

[14]. Lee $\mathrm{MH}$, Haut RC. "Insensitivity and tensile breakdown properties ofthe human parasagittal bridging veins to strain rate dependence inbiomechanics of subdural hematoma".JBiom. 1989;22:532-542.

[15]. Lowenhielm P. "Dynamic properties of parasagittal bridging veins."'ZRechtsmed. 1974;74:55-62.

[16]. Lubock P, Goldsmith W. "Experimental cavitation studies in a model head-neck system.”JBiomech.

[17]. 1980; 13(12):1041-1052.

[18]. Moss, William C., Michael J. King, and Eric G. Blackman. "Skull flexure from blast waves: a mechanism for brain injury with implications for helmet design."Physical review letters 103.10 (2009): 108702.

[19]. Goeller, Jacques, et al. "Investigation of cavitation as a possible damage mechanism in blast-induced traumatic brain injury." Journal of neurotrauma29.10 (2012): 1970-1981.

[20]. Ommaya, Ayub K., Robert L. Grubb Jr, and Ronald A. Naumann. "Coup and contre-coup injury: observations on the mechanics of visible brain injuries in the rhesus monkey." Journal of Neurosurgery 35.5 (1971): 503-516.

[21]. Sundaramurthy, Aravind, et al. "Blast-induced biomechanical loading of the rat: an experimental and anatomically accurate computational blast injury model."Journal of neurotrauma 29.13 (2012): 2352-2364.

[22]. Viano DC. 1988. "Cause and control of automotive trauma." Bull NY Acad Med 64:376.

[23]. Viano, David C., and Albert I. King."Biomechanics of chest and abdomen impact." The biomedical engineering handbook 1 (2000): 369-380. 